Western University

Scholarship@Western

Chemistry Publications

Chemistry Department

11-19-2009

Increased yields and simplified purification with a second-generation cobalt catalyst for the oxidative formation of trans-THF rings.

Cory Palmer

Nicholas A Morra

Andrew C Stevens

Barbora Bajtos

Ben P Machin

See next page for additional authors

Follow this and additional works at: https://ir.lib.uwo.ca/chempub

Part of the Chemistry Commons

Citation of this paper:

Palmer, Cory; Morra, Nicholas A; Stevens, Andrew C; Bajtos, Barbora; Machin, Ben P; and Pagenkopf, Brian L, "Increased yields and simplified purification with a second-generation cobalt catalyst for the oxidative formation of trans-THF rings." (2009). Chemistry Publications. 50.

https://ir.lib.uwo.ca/chempub/50 
Authors

Cory Palmer, Nicholas A Morra, Andrew C Stevens, Barbora Bajtos, Ben P Machin, and Brian L Pagenkopf 


\title{
Increased Yields and Simplified Purification with a Second Generation Cobalt Catalyst for the Oxidative Formation of trans- THF Rings
}

\author{
Cory Palmer, Nicholas A. Morra, Andrew C. Stevens, Barbora \\ Bajtos, Ben P. Machin, and Brian L. Pagenkopf*
}

The University of Western Ontario, Department of Chemistry, London, Ontario, N6A

$5 B 7$

bpagenko@uwo.ca

Received Date (will be automatically inserted after manuscript is accepted)

\section{ABSTRACT}

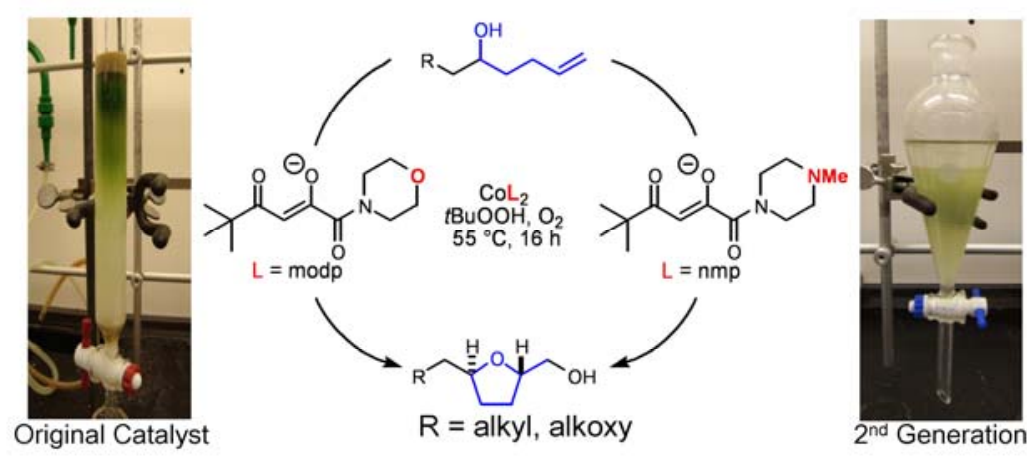

The synthesis of a second generation cobalt catalyst for the formation of trans-THF products via the Mukaiyama aerobic oxidative cyclization is reported. Two procedures have been developed with the new water-soluble catalyst that give superior yields and greatly simplify purification compared to the previous catalysts.

The ubiquitous nature of tetrahydrofuran (THF) rings in a wide variety of biologically active natural products has inspired the development of methods for their synthesis and derivatization. ${ }^{1}$ In particular, the ability to form 2,5-trans-THF rings in an efficient and diastereoselective manner is essential for the synthesis of many natural products displaying this structural motif. Many methods have been utilized to access trans-THF rings. However, some methods have poor yields or low

(1) (a) Bermejo, A.; Figadere, B.; Zafra-Polo, M.-C.; Barrachina, I.; Estornell, E.; Cortes, D. Nat. Prod. Rep. 2005, 22, 269-303. (b) Kobayashi, J.; Kubota, T. J. Nat. Prod. 2007, 70, 451-460. (c) Kobayashi, J. J. Antiobiotics 2008, 61, 271-284. diastereoselectivities. $^{2}$ The Mukaiyama aerobic oxidative cyclization is emerging as a powerful synthetic tool that uses molecular oxygen as the stoichiometric oxidant to convert pentenols to trans-THF rings (Scheme 1$)^{3}$ The reaction has been the subject of mechanistic investigations, ${ }^{4}$ and has also been utilized in total

(2) For reviews see: (a) Cardillo, G.; Orena, M. Tetrahedron 1990, 46, 3321-3408. (b) Wolfe, J. P.; Hay, M. B. Tetrahedron 2007, 63, 261290. (c) Li, N.; Shi, Z.; Tang, T.; Chen, J.; Li, X. Beilstein J. Org. Chem. 2008, 4.

(3) Inoki, S,; Mukaiyama, T. Chem. Lett. 1990, 1, 67-70.

(4) (a) Pérez, B. M.; Schuch, D.; Hartung, J. Org. Biomol. Chem. 2008, 6, 3532-3541. (b) Schuch, D.; Fries, P.; Donges, M.; Pérez, B.M.; Hartung, J. Am. Chem. Soc. 2009, 131, 12918-12920 
synthesis (Figure 1). ${ }^{5}$ Our research group has applied this strategy in the total synthesis of aplysiallene ${ }^{6}$ and bullatacin. ${ }^{7}$ Herein, we report our success in the development of a second generation cobalt catalyst for the formation of trans-THF rings that gives superior yields and greatly simplifies purification when compared to the standard catalysts.

Scheme 1. A Representative Mukaiyama Aerobic Oxidative Cyclization
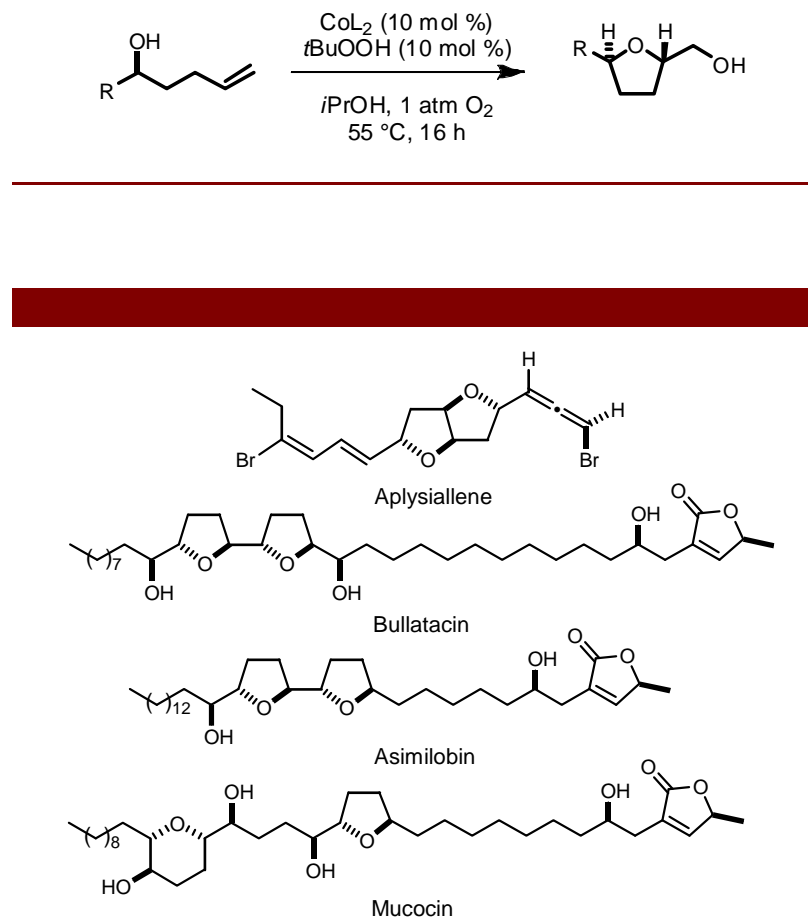

Figure 1. Natural products containing trans-THF rings synthesized using the Mukaiyama oxidation ${ }^{8}$

The Mukaiyama oxidation typically proceeds in good yield and excellent diastereoselectivity ( $>99: 1 \mathrm{dr}$ ); however, in some cases the catalyst was extraordinarily difficult to remove from the desired THF product when the standard ligands $\mathbf{1}$ and $\mathbf{2}$ (Figure 2) were utilized. ${ }^{9}$ Furthermore, paramagnetic cobalt residues interfere with NMR analysis, making characterization of the cyclized

(5) (a) Wang, Z.-M.; Tian, S.-K..; Shi, M.; Tetrahedron Lett. 1999, 40, 997-980. (b) Tian, S-K.; Wang, Z-M.; Jiang, J-K.; Shi, M. Tetrahedron: Asymmetry 1999, 10, 2551-2562. (c) Wang, Z.-M.; Tian, S.-K.; Shi, M. Tetrahedron: Asymmetry 1999, 10, 667-670 (d) Wang, Z.-M.; Tian, S.-K.; Shi, M. Eur. J. Org. Chem. 2000, 2, 349-356. (e). Wang, Z.-M.; Tian, S.-K.; Shi, M. Chirality 2000, 12, 581-587. (f) Evans, P.A.; Cui, J.; Gharpure, S. J.; Polosukhin, A.; Zhang, H.-R. J. Am. Chem. Soc. 2003, 125, 14702-14703.

(6) Wang, J.; Pagenkopf, B. L. Org. Lett. 2007, 9, 3703-3706.

(7) Zhao, H.; Gorman, J. S. T.; Pagenkopf, B. L. Org. Lett. 2006, 8, 4379-4382.

(8) For aplysiallene, see ref 6. For bullatacin, see ref 7. For asimilobin, see ref $5 \mathrm{a}$. For mucocin, see ref $5 \mathrm{f}$.

(9) See supporting information for photographs showing elution of cobalt residues. product difficult or impossible. The difficulties associated with purification detract from the synthetic advantages of the catalyst. Thus, we set out to prepare a new catalyst that retains high efficiency, but also exhibits significantly increased polarity. This and related strategies have seen great success with EDC, water soluble ligands, sulphonated phosphines, fluorous phases and ionic liquids. ${ }^{10}$ Using the well established modp ligand $\mathbf{1}$ as a template, it was envisioned that replacing the neutral morpholine subunit with a basic $N$-methyl piperazine (3, 5,5-dimethyl-1-(4-methylpiperazin-1yl)hexane-1,2,4-trione, henceforth $\mathrm{nmp}$ ) might facilitate removal of the cobalt species from the reaction mixture by a straightforward acidic extraction.

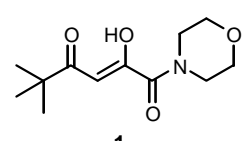

1

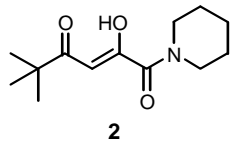

2

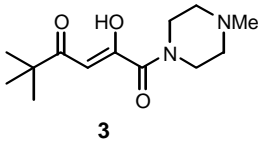

Figure 2. Commonly used modp (1) and piper (2) ligands, and water soluble nmp (3)

The synthesis of the nmp ligand commenced with reaction of $N$-methyl piperazine with ethyl oxalyl chloride in the presence of triethylamine, which proceeded in near quantitative yield (Scheme 2). A significant loss of product was observed (ca. 50\%) when the subsequent Claisen condensation of $\mathbf{4}$ with pinacolone was subjected to a standard aqueous workup. ${ }^{7}$ Fortuitously, an alternative protonation method employing acetic acid in $\mathrm{CH}_{2} \mathrm{Cl}_{2}$ followed by removal of the salts by filtration afforded ligand $\mathbf{3}$ in 95\% yield. Complexation of the nmp ligand 3 with Co(ethyl-2hexanoate $)_{2}$ in dry benzene was initially low yielding and the resulting purple-colored solid performed poorly in subsequent cyclization reactions. Previously, we reported the first X-ray structures of these types of catalysts that clearly showed adventitious water in the crystal structure. ${ }^{11}$ Complexation in aqueous benzene provided $\mathrm{Co}(\mathrm{nmp})_{2}$ (5) as a tan coloured solid in $89 \%$ yield over three steps, using bench top centrifugation as the only method of purification to collect the catalyst. Gratifyingly, the oxidation results with the $\mathrm{Co}(\mathrm{nmp})_{2}$ catalyst formed under aqueous conditions proved to be exceptional (vide infra).

(10) (a) Sheehan, J. C.; Cruickshank, P. A.; Boshart, G. L. J. Org. Chem. 1961, 26, 2525-2528. (b) ) Herrmann, W. A.; Kulpe, J. A.; Konkol, W.; Bahrmann, H. J. Organomet. Chem. 1990, 389, 1, 85-101. (c) Water soluble ligands. Herrmann, W. A.; Herrmann, W. A.; Kohlpaintner, C. W. Angew. Chem. Int. Ed. Engl. 1993, 32, 1524-1544. (d) Curran, D. P. Aldrichimica Acta 2006, 39, 3-9.

(11) Wang, J.; Morra, N. A.; Zhao, H.; Gorman, J.S.T.; Lynch, V.; McDonald, R.; Reichwein, J.F.; Pagenkopf, B. L. Can. J. Chem. 2009, 87, 328-334. The actual molecular formula is most likely $\left[\left\{\mathrm{Co}(\mathrm{nmp})_{3}\right\}_{2} \mathrm{Co}\left(\mathrm{H}_{2} \mathrm{O}\right)_{6}\right]$, but is represented as $\mathrm{Co}(\mathrm{nmp})_{2}$ for simplicity. 
Scheme 2. Synthesis of $\operatorname{Co}(n m p)_{2}$<smiles></smiles>

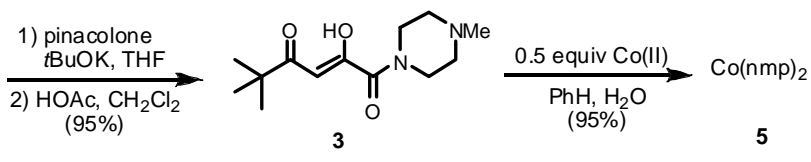

To compare the new $\operatorname{Co}(\mathrm{nmp})_{2}$ catalyst to previous ones $\left(\mathrm{Co}(\operatorname{modp})_{2}\right.$ and $\left.\mathrm{Co}(\text { piper })_{2}\right)$, a family of TBSprotected pentenol derivatives that have proven to be reliable substrates in these reactions were selected (Table 1). Encouragingly, these early results with $\operatorname{Co}(\mathrm{nmp})_{2}$ demonstrated a remarkable improvement in the overall yield of the oxidative cyclization process. These new conditions also circumvent the undesired side reactions leading to aldehydes and protocyclization products, typically observed with traditional catalysts. ${ }^{2}$

Table 1. Initial Comparison of the Catalysts

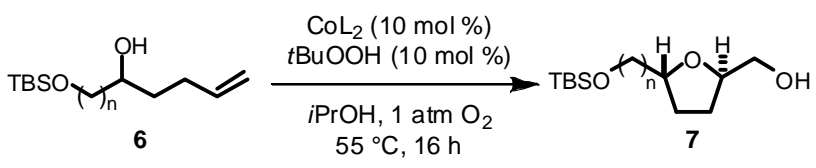

\begin{tabular}{ccccccc}
\hline & & \multicolumn{5}{c}{ yield (\%) } \\
\cline { 5 - 7 } entry & $\begin{array}{c}\text { starting } \\
\text { material }\end{array}$ & $\mathrm{n}$ & modp & piper & $\begin{array}{c}\text { nmp } \\
\text { buffer }^{a}\end{array}$ & $\begin{array}{c}\text { nmp } \\
\text { MeI }\end{array}$ \\
\hline 1 & $\mathbf{6 a}$ & 1 & 65 & 39 & 92 & 93 \\
2 & $\mathbf{6 b}$ & 2 & 68 & 75 & 90 & 90 \\
3 & $\mathbf{6 c}$ & 3 & 79 & 74 & 92 & 95 \\
${ }^{2} \mathrm{pH}$ & 4 & & & & \\
\hline
\end{tabular}

With regards to product purification, it is important to note that when using the $\operatorname{Co}(\mathrm{nmp})_{2}$ catalyst system complete removal of the cobalt residues from the transTHF products was achieved easily with $\mathrm{pH} 4$ phosphate buffer wash of the organic layer. Anticipating the need for a neutral workup procedure to remove the catalyst from more acid sensitive substrates, the tertiary amine was quaternized by treating the reaction mixture with MeI under an argon atmosphere. The resulting salts are highly water soluble and can be removed using a simple neutral water extraction. The new $\mathrm{Co}(\mathrm{nmp})_{2}$ catalyst system facilitates a mild and straightforward isolation and purification process, and when coupled with the improved yields, it greatly enhances the synthetic potential of this methodology.

The effect of catalyst loading on cyclization efficiency was investigated (Table 2). The typical catalyst loading reported for $\mathrm{Co}(\operatorname{modp})_{2}$ cyclizations is $10 \mathrm{~mol} \%$, and in the oxidation of $\mathbf{6 b}$ (Table 2) the yield did not improve significantly at higher loading (entry 1), while with lower catalyst loading the yield dropped significantly (entry 3). Gratifyingly, it was observed that reactions using Co(nmp) 2 proceeded efficiently with catalyst loadings as low as $5 \mathrm{~mol} \%$ (entries $4-6$ ), while lower loadings resulted in incomplete conversion (entries $7-8$ ). It is noteworthy that with $\mathrm{Co}(\operatorname{modp})_{2}$ at $5 \mathrm{~mol} \%$ catalyst loading byproduct formation becomes seriously competitive, but these side reactions are not observed with catalyst loading as low as $3 \mathrm{~mol} \%$ with the new $\mathrm{Co}(\mathrm{nmp})_{2}$ system (compare entries 3 with 6 and 7).

Table 2. Effect of Catalyst Loadings on the Oxidation of $\mathbf{6 b}$

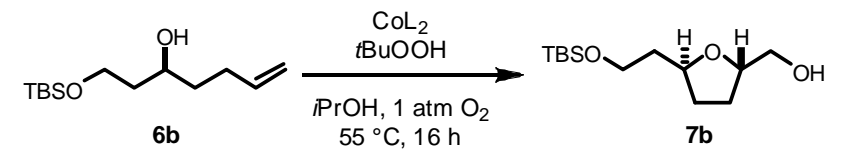

\begin{tabular}{cccc}
\hline entry & catalyst & mol \% & yield (\%) \\
\hline 1 & $\mathrm{Co}(\text { modp })_{2}$ & 15 & 68 \\
2 & $\mathrm{Co}(\text { modp })_{2}$ & 10 & 65 \\
3 & $\mathrm{Co}(\text { modp })_{2}$ & 5 & $47^{\mathrm{a}}$ \\
4 & $\mathrm{Co}(\mathrm{nmp})_{2}$ & 15 & 95 \\
5 & $\mathrm{Co}(\mathrm{nmp})_{2}$ & 10 & 97 \\
6 & $\mathrm{Co}(\mathrm{nmp})_{2}$ & 5 & 93 \\
7 & $\mathrm{Co}(\mathrm{nmp})_{2}$ & 3 & $57(93)^{b}$ \\
8 & $\mathrm{Co}(\mathrm{nmp})_{2}$ & 1 & $10(77)^{b}$
\end{tabular}

${ }^{a}$ All starting material was consumed. ${ }^{b}$ Yield based on recovered starting material.

Encouraged by the results with $\mathrm{Co}(\mathrm{nmp})_{2}$ in the initial screening of TBS protected pentenol derivatives, its performance was compared against the standard cobalt catalysts with a variety of additional substrates (Table 3). In all cases examined, reactions run with the new $\mathrm{Co}(\mathrm{nmp})_{2}$ catalyst resulted in an increased yield compared to the original catalyst. The polar hydrophilic THF (entry

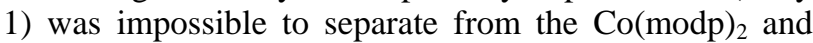
$\mathrm{Co}$ (piper) $)_{2}$ catalyst residues even after repeated column chromatography with different eluents. In contrast, after in situ methylation of the $\mathrm{Co}(\mathrm{nmp})_{2}$ species, the THF product was obtained in pure form in $85 \%$ yield after an aqueous extraction. The results in entries 2 and 3 were equally satisfying, and it is noteworthy that the oxidatively labile benzyl and p-methoxyphenyl ether protecting groups survive the reaction conditions. ${ }^{12} \mathrm{~A}$ symmetric pentadienol (entry 4), in addition to sterically

(12) Previous studies in our laboratory have shown that PMB protective groups are destructively cleaved under the conditions of the oxidative cyclization. 
encumbered substrates (entries 5 and 6) were also found to readily cyclize under the oxidation conditions. Furthermore, aromatic substrates including phenyl, benzyl, and cinnamic acid derivatives all gave favorable results (entries $6-9$, respectively). Finally, the compounds in entries 10 and 11 are intermediates that were employed in the total synthesis of aplysiallene ${ }^{6}$ and bullatacin, ${ }^{7}$ respectively, thus further illustrating the overall superiority and utility of this new catalyst for natural product synthesis.

Table 3. Cyclization Precursors and Their Respective Cyclized Products

\begin{tabular}{|c|c|c|c|c|c|c|}
\hline \multirow{3}{*}{ entry ${ }^{a}$} & \multirow{3}{*}{ starting Material } & \multirow{3}{*}{ product } & \multicolumn{4}{|c|}{ yield (\%) } \\
\hline & & & \multirow{2}{*}{$\mathrm{Co}(\text { piper })_{2}$} & \multirow{2}{*}{$\mathrm{Co}(\operatorname{modp})_{2}$} & \multicolumn{2}{|c|}{$\mathrm{Co}(\mathrm{nmp})_{2}$} \\
\hline & & & & & MeI & buffer \\
\hline $1^{b}$ & & & $69^{d}$ & $64^{d}$ & 85 & 54 \\
\hline $2^{b}$ & & & 20 & 58 & 90 & 66 \\
\hline $3^{b}$ & & & 29 & $81^{d}$ & 90 & 85 \\
\hline 4 & & & 46 & 57 & 95 & 40 \\
\hline 5 & & & 62 & 76 & 91 & 58 \\
\hline 6 & & & 54 & 47 & 91 & 45 \\
\hline 7 & & & 52 & 70 & 90 & 60 \\
\hline 8 & & & $71^{d}$ & 49 & 86 & 80 \\
\hline 9 & & & 38 & 42 & 88 & 46 \\
\hline $10^{c}$ & & & 55 & $81^{e}$ & 83 & 78 \\
\hline $11^{c}$ & & & 69 & $79^{f}$ & 88 & 79 \\
\hline
\end{tabular}

In summary, a second generation catalyst for the Mukaiyama oxidative cyclization is reported. The catalyst preparation is straightforward at gram scale with centrifugation as the only means of purification. In all cases examined, cyclization reactions using $\mathrm{Co}(\mathrm{nmp})_{2}$ have shown improved yields and fewer side products. Most importantly, Co(nmp) $)_{2}$ has greatly simplified postreaction purification by replacing difficult column chromatography with an aqueous workup. We believe this new second generation catalyst now clearly stands among the best methods to prepare 2,5-disubstituted trans-THF rings and it is sure to find applications in total synthesis.
Acknowledgment We thank the University of Western Ontario and the National Sciences and Engineering Research Counsil of Canada for financial asistance. NM (NSERC CGS D) and BB (Vanier CGS) thank NSERC for their graduate fellowships. We also thank James Russell Macdonald for experimental assistance.

Supporting Information Available Experimental procedures and characterization of all new compounds and copies of NMR spectra. This material is available free of charge via the Internet at http://pubs.acs.org. 